\section{MR Imaging Findings in Wernicke Encephalopathy: Nonalcoholics May Be Similar to Alcoholics}

We read with special interest the article of Zuccoli et al ${ }^{1}$ entitled "MR Imaging Findings in 56 Patients with Wernicke Encephalopathy: Nonalcoholics May Differ From Alcoholics." The authors reported the typical and atypical imaging findings of Wernicke encephalopathy (WE). They suggested that alcoholic and nonalcoholic patients present different MR imaging patterns due to differences in the pathophysiologic process. Typical imaging findings, which were more common in alcoholic patients, consist of symmetric signal-intensity abnormalities in the thalami, mamillary bodies, tectal plate, and periaqueductal area, as well as contrast enhancement in the mamillary bodies and thalami, whereas signal-intensity alterations in the cerebellum, cerebral vermis, cranial nerve nuclei, red nuclei, dentate nuclei, caudate nuclei, splenium, and cerebral cortex represent atypical MR imaging findings and are more frequent in nonalcoholic patients. The authors speculated that alcohol might have a protective effect on the brain areas that show atypical lesions in WE, which could explain why atypical findings are seen only in nonalcoholic patients.

We report 2 cases of nonalcoholic WE: 1 presenting with typical and another with atypical MR imaging findings. The first case is a 21-year-old woman with non-Hodgkin lymphoma, having undergone chemotherapy and bone marrow transplant (BMT). Thirty-one days after BMT and receiving parenteral nutrition, she presented neurologic and visual disturbances first attributed to cyclosporine. However, MR imaging findings revealed, on T2-weighted images, high signal intensity symmetrically and bilaterally in the medial vestibular nuclei, facial nuclei, medial and pulvinar thalami (Fig 1A), and periventricular region of the third ventricle, as well as in the mamillary bodies. After gadolinium administration, there was enhancement in the mamillary bodies and in the facial nuclei (Fig 1B).

The additional case was a 13 -year-old boy with suppurative appendicitis, who underwent laparotomy complicated with mesenteric infarction, adhesion, and hematoma of the rectum. Thus, he underwent a colostomy, and after a month of parenteral nutrition, he developed a sixth-nerve palsy, seizures, and coma. The MR imaging demonstrated well-defined symmetric and bilateral lesions hyperintense on T2-weighted and fluid-attenuated inversion recovery (FLAIR) images in the tectum of the midbrain, the periaqueductal gray matter, mamillary bodies, thalami, putamina, and periventricular region of the third ventricle, as well as in the frontal cortex. The lesions also presented restricted diffusion and no contrast enhancement. In both cases, the diagnosis of WE was suggested and appropriate therapy was initiated. However, the patients did not respond and died in the following days.
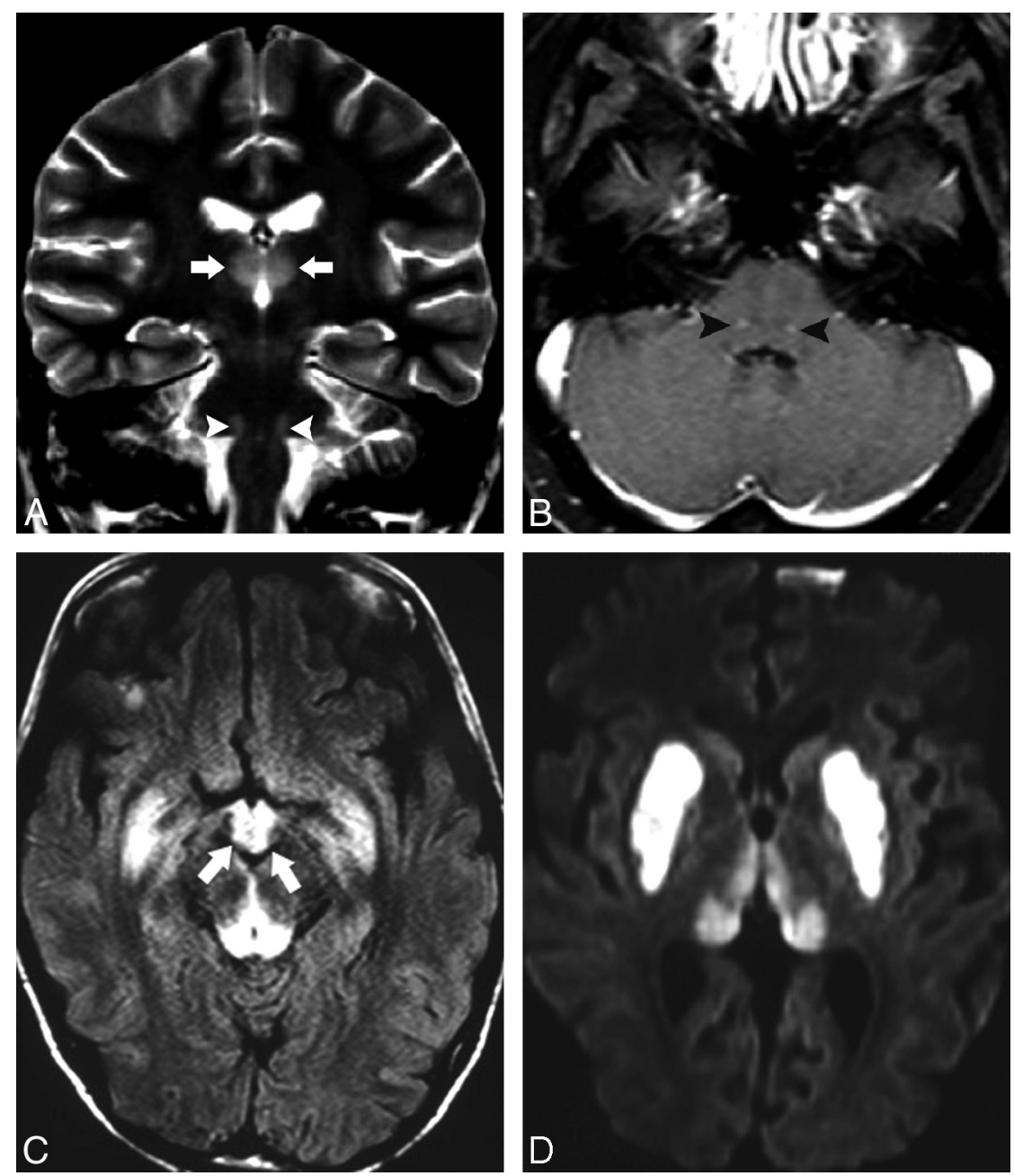

Fig 1. Case 1. Coronal T2-weighted image $(A)$ shows high signal intensity in the thalami (arrows) and facial nuclei (arrowheads), which demonstrates contrast enhancement (arrowheads) in the T1-weighted image $(B)$ after gadolinium administration. Case 2. FLAIR images ( $C$ and $D$ ) show bilateral and symmetric areas of high signal intensity on the mamillary bodies (arrows), periaqueductal gray matter, thalami, and putamina. 
We report these cases of nonalcoholic WE demonstrating that both typical and atypical MR imaging findings may coexist. Although Zuccoli et al ${ }^{1}$ suggested that contrast enhancement of the mamillary bodies and thalami indicate alcoholic WE findings, these findings were already demonstrated in nonalcoholic patients, ${ }^{2}$ corroborating our cases. Our findings indicate that the WE physiopathology might not differ between alcoholic and nonalcoholic patients. Alcohol may not play as important a role as thiamine deficiency does in the whole process. Many clinical conditions can directly or indirectly cause thiamine deficiency, which will lead to WE.

Finally, as suggested by Zuccoli et al, ${ }^{1,3}$ the correct diagnosis is the main challenge in the clinical approach to these patients. The initiation of the required treatment can avoid or delay the appearance of severe neurologic deficits and even death, no matter the cause of thiamine deficiency.

\section{References}

1. Zuccoli G, Santa Cruz D, Bertolini M, et al. MR imaging findings in 56 patients with Wernicke encephalopathy: nonalcoholics may differ from alcoholics. AJNR Am J Neuroradiol 2009;30:171-76. Epub 2008 Oct 22

2. Fei GQ, Zhong $\mathrm{C}$, Jin $\mathrm{L}$, et al. Clinical characteristics and MR imaging features of nonalcoholic Wernicke encephalopathy. AJNR Am J Neuroradiol 2008;29:164-69

3. Zuccoli G, Gallucci M, Capellades J, et al. Wernicke encephalopathy: MR findings in twenty-six alcoholic and non-alcoholic patients. AJNR Am J Neuroradiol 2007;28:1328-31

L.C. Hygino da Cruz, Jr

R.C. Domingues

Clínica de Diagnóstico Por Imagem

I. Vilanova

Clinic IRM

E.L. Gasparetto

Department of Radiology

University Federal of Rio de Janeiro

Rio de Janeiro, Brazil

DOI 10.3174/ajnr.A2113 